\title{
Récidives de mycétome après amputation à Dakar (Sénégal)
}

\section{Recidives of Mycetoma after Amputation in Dakar (Senegal)}

\author{
L. Sarr · B. Dembélé · M. Limam · M. Daffé - AB Diouf · AB Gueye · S. Diao · M. Diop · N.F. Coulibaly $\cdot$ C. Diémé \\ Reçu le 11 avril 2019; accepté le 12 décembre 2019 \\ (C) Société de pathologie exotique et Lavoisier SAS 2019
}

Résumé La chirurgie constitue le temps essentiel du traitement des mycétomes fongiques. Elle consiste en une amputation en cas d'atteinte osseuse. Nous avons observé 5 cas de récidives après amputation pour mycétome. Il s'agit dans tous les cas de patients présentant des mycétomes à grain noir avec atteintes osseuses. Les récidives sont survenues à moins de 18 mois de l'amputation faisant parler de reprise évolutive et posant le problème du niveau de l'amputation.

Mots clés Récidive · Amputation · Mycétomef · Hôpital · Mauritanie $\cdot$ Sénégal $\cdot$ Afrique

Summary The treatment of fungal mycetoma is essentially surgical. This carcinological-like surgery consists of amputation in case of bone involvement. The recurrences after amputation are rare and address the problem of the operative indication. We report 5 cases of recurrence of fungal blackgrain mycetoma after amputation of leg or thigh.

Case 1: a 52-year-old patient with a mycetoma of the knee evolving for 8 years. There is no history of surgery. A thigh amputation with ganglion dissection is performed. One year after the surgical procedure, the patient presents a recurrence on the amputation stump and on the lymph node dissection

L. Sarr $(\bowtie) \cdot$ B. Dembélé · M. Daffé · AB Diouf · M. Diop N.F. Coulibaly · C. Diémé

Service orthopédie-traumatologie, Hôpital Aristide Le Dantec, Dakar, Sénégal

e-mail : drlaminesarr@yahoo.fr

S. Diao

Service orthopédie-traumatologie, Hôpital Général de Grand

Yoff, Dakar, Sénégal

AB Gueye

Centre hospitalier de l'Ordre de Malte, Dakar, Sénégal

M. Limam

Service d'orthopédie-traumatologie, Hôpital Cheikh Zayed, Nouakchott, Mauritanie site. An indication of hip disarticulation is made and performed 17 months after amputation.

Case 2: a 25-year-old patient who has a black-grain mycetoma of the foot with osteitis evolving since 10 years. A leg amputation was performed. The patient had a recurrence at the popliteal level at 15 months postoperatively. An indication of amputation of the thigh is posed and refused by the patient.

Case 3: a30-year-old woman with black-grain mycetoma of the knee with bone involvement for more than 10 years. A thigh amputation was performed and at nine months postoperativeshe presented a recurrence in the amputation stump. She was lost of sight despite the decision of surgical revision.

Case 4: a 43-year-old patient operated on his foot and leg mycetoma at least 5 timesbefore amputation in 2000. The recurrence occurred one year after amputation. 18 years after amputation, a new surgical procedure was difficult due to extension of the lesions in the pelvis.

Case 5: a 50-year-old female patient operated in Mauritania in 2012 (thigh amputation for mycetoma of the knee). She presented a recurrence on the amputation stump in 2018. An indication of disarticulation of the hip was posed and refused by the patient.

These recurrences were testified by to the persistence of grains on the preserved segment. They pose the problem of the level of amputation and therefore of preoperative planning. Good preoperative planning allows optimization of the surgical procedure and avoids certain recurrences.

Keywords Recurrence - Amputation - Fungal Mycetoma . Hospital $\cdot$ Mauritania $\cdot$ Senegal $\cdot$ Africa

\section{Introduction}

Le traitement des mycétomes fongiques est essentiellement chirurgical. Il s'agit d'une chirurgie de type carcinologique consistant en une exérèse ou en une amputation en cas 
d'atteinte osseuse. Les récidives après amputation sont rares et posent le problème de l'indication opératoire.

Nous rapportons cinq cas de récidives de mycétomes fongiques à grain noir après amputation à partir desquels nous analysons les facteurs qui prédisposent à ces récidives.

\section{Cas cliniques}

\section{Cas 1 (Fig. 1,2,3)}

M. S., cultivateur et éleveur de 52 ans, originaire du nord du Sénégal à la frontière avec la Mauritanie a été reçu pour un mycétome du genou à grains noirs évoluant depuis plus de 8 ans. Une première exérèse a été réalisée en 2010 dans un hôpital régional. En novembre 2016, il présentait à l'examen des fistules postéro-médiales au-dessus du creux poplité. L'imagerie a permis de mettre en évidence une ostéolyse métaphysaire distale du fémur avec présence sur les coupes scannographiques de petites lacunes le long de la diaphyse fémorale, témoin d'une extension à l'image des skip métastases des tumeurs. Une amputation de cuisse avec curage ganglionnaire a été réalisée en février 2017.

À M12 postopératoire, on notait une fistulisation avec issue de grains noirs sur la cicatrice opératoire et sur le site du curage ganglionnaire témoin d'une récidive. La radiographie standard permettait de noter une ostéite du moignon d'amputation. Une indication de désarticulation de hanche a été posée et réalisée 17 mois après l'amputation.

\section{Cas 2 (Fig. 4)}

A.B., patient de 25 ans, présentait un mycétome du pied à grain noir avec ostéite évoluant depuis 10 ans, associée à une masse du creux poplité sans atteinte osseuse. Une amputation de jambe avec exérèse de la masse du creux poplité a été réalisée. L'évolution a été marquée par une récidive au niveau du creux poplité à 15 mois postopératoire. Une indication d'amputation de cuisse a été posée et refusée par le patient.

\section{Cas 3 (Fig. 5)}

M.S., patiente de 30 ans présentait un mycétome à grains noirs du genou évoluant depuis plus de 10 ans avec atteinte osseuse. Une amputation de cuisse a été réalisée. Elle a été revue à plus de 16 mois après l'opération et présentait une récidive au niveau du moignon d'amputation. Une désarticulation de hanche a été refusée par la patiente. Elle a été également perdue de vue.

\section{Cas 4 (Fig. 6)}

F. K., patient de 43 ans, opéré plus de 5 fois pour mycétome du pied à grains noirs, venait de la région de Saint Louis dans le nord du Sénégal. La notion de piqûre par épine d'arbuste n'a pas été rapportée. La durée d'évolution de la symptomatologie avant la première intervention, qui remonte à l'année 1990, n'est pas connue par le patient. Il a présenté de multiples récidives avec extension au niveau du genou et des adénopathies inguinales. L'amputation de la cuisse a été faite en 2001. Une récidive s'est vite installée (moins d'une année après l'amputation). À l'examen, 18 ans après son amputation, il présentait de multiples fistules au niveau du moignon d'amputation, également à la face antérieure de la hanche. Nous n'avons pas voulu refaire un geste chirurgical, vue l'extension des lésions au bassin.
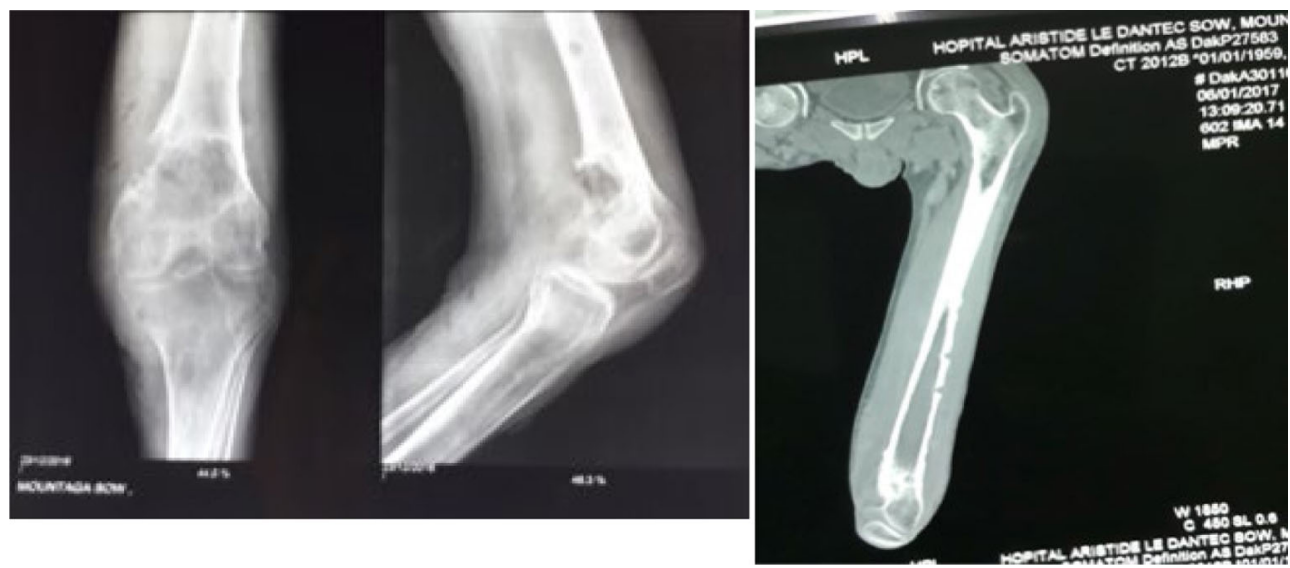

Fig. 1 Lyse métaphysaire distale du fémur avec lacunes corticales étagées sur la diaphyse à l'image des skip métastases des tumeurs / Distal metaphyseal lysis of the femur with cortical gaps staggered on the diaphysis like skip metastases of tumors. 


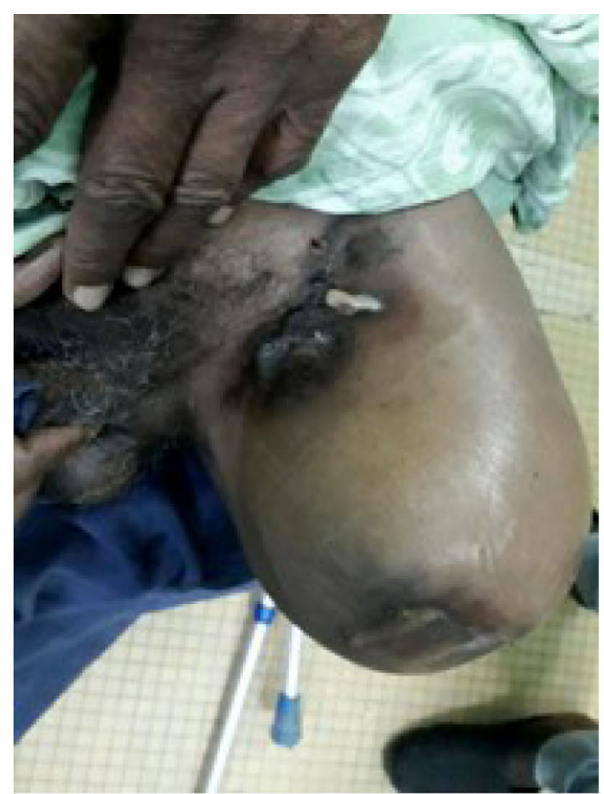

a)

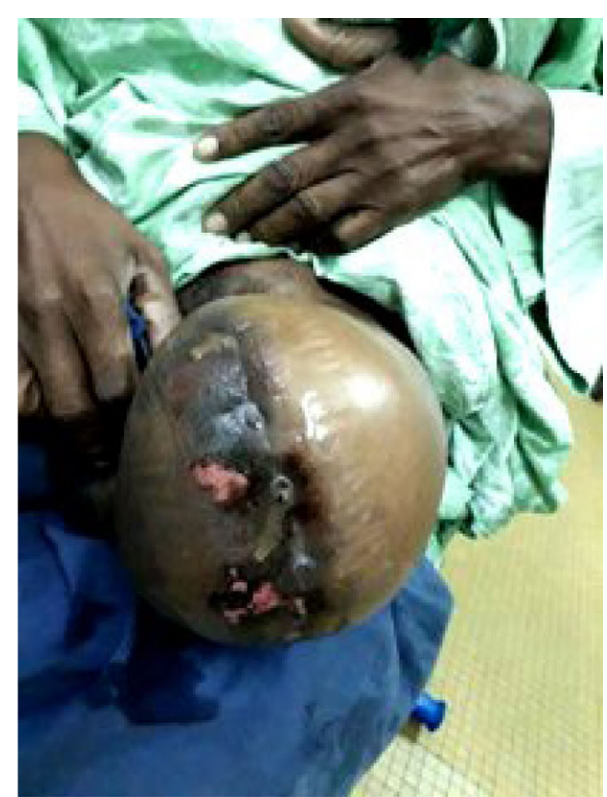

b)

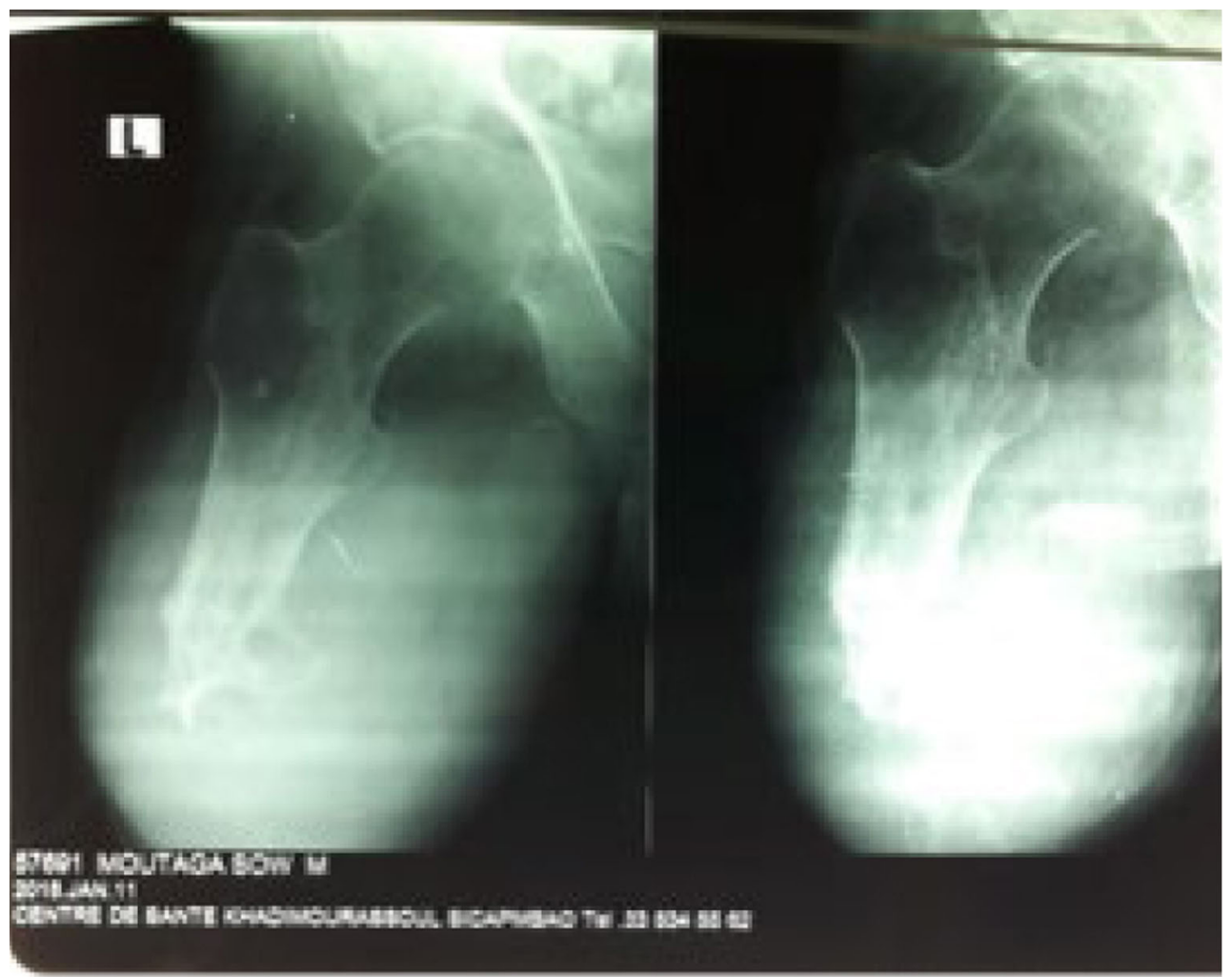

Fig. 2 a) Fistules inguinales et de la cicatrice du moignon d'amputation avec issue de pus franc et de grains noirs ; b) Ostéite du moignon $\mathrm{d}$ 'amputation / a) Inguinal fistulas and amputation stump scar with discharge of purulent fluid and black grains ; b) Osteitis of the amputation stump

\section{Cas 5 (Fig. 7)}

Il s'agit d'une femme au foyer de 50 ans, opérée en Mauritanie en 2012 par une mission étrangère pour mycétome du genou, évoluant depuis plus de 10 ans, pour lequel une amputation de cuisse a été réalisée.

La récidive du mycétome a été diagnostiquée à Nouakchott en 2018, soit 6 ans après l'amputation. La patiente 


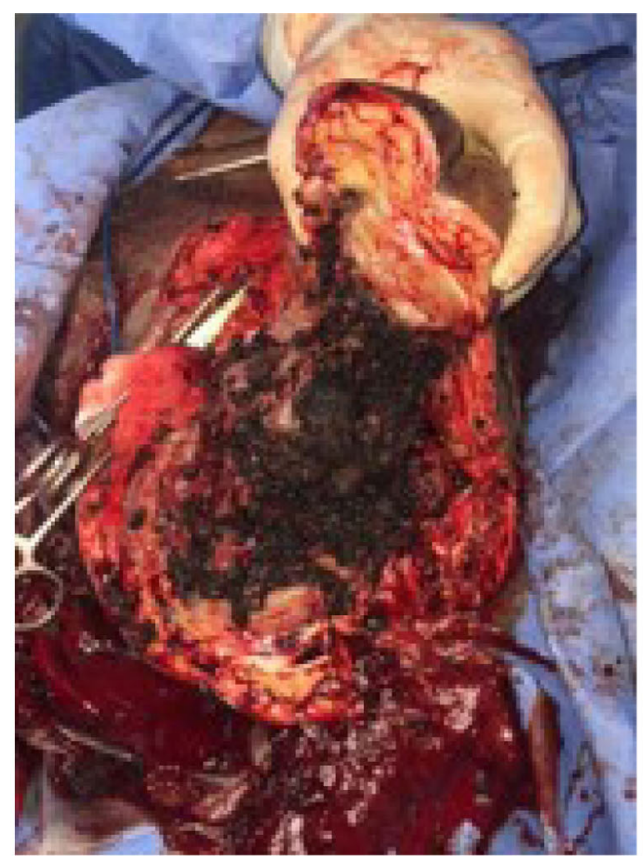

a)

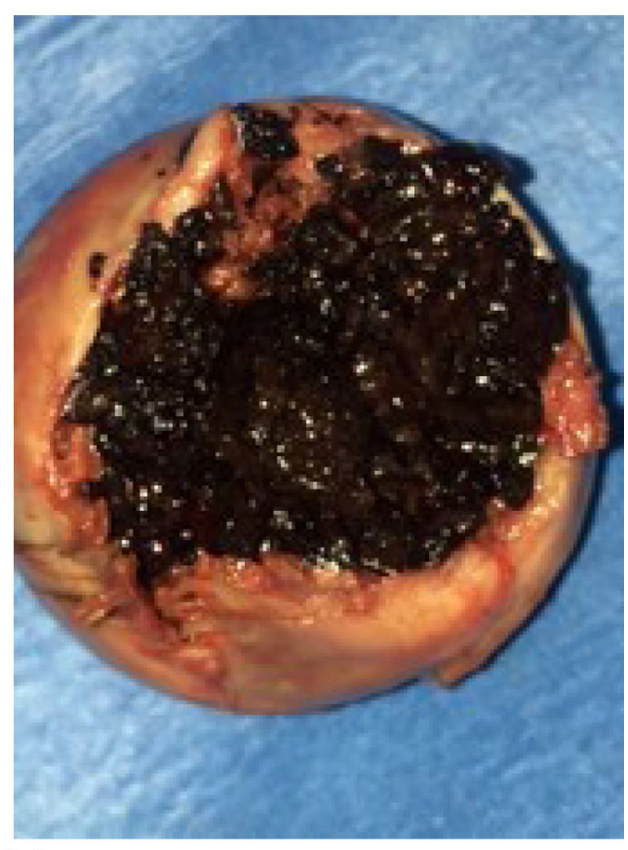

b)

Fig. 3 Importante quantité de grains lors de la désarticulation et sur la tête fémorale / Significant amount of grains during disarticulation and on the femoral head

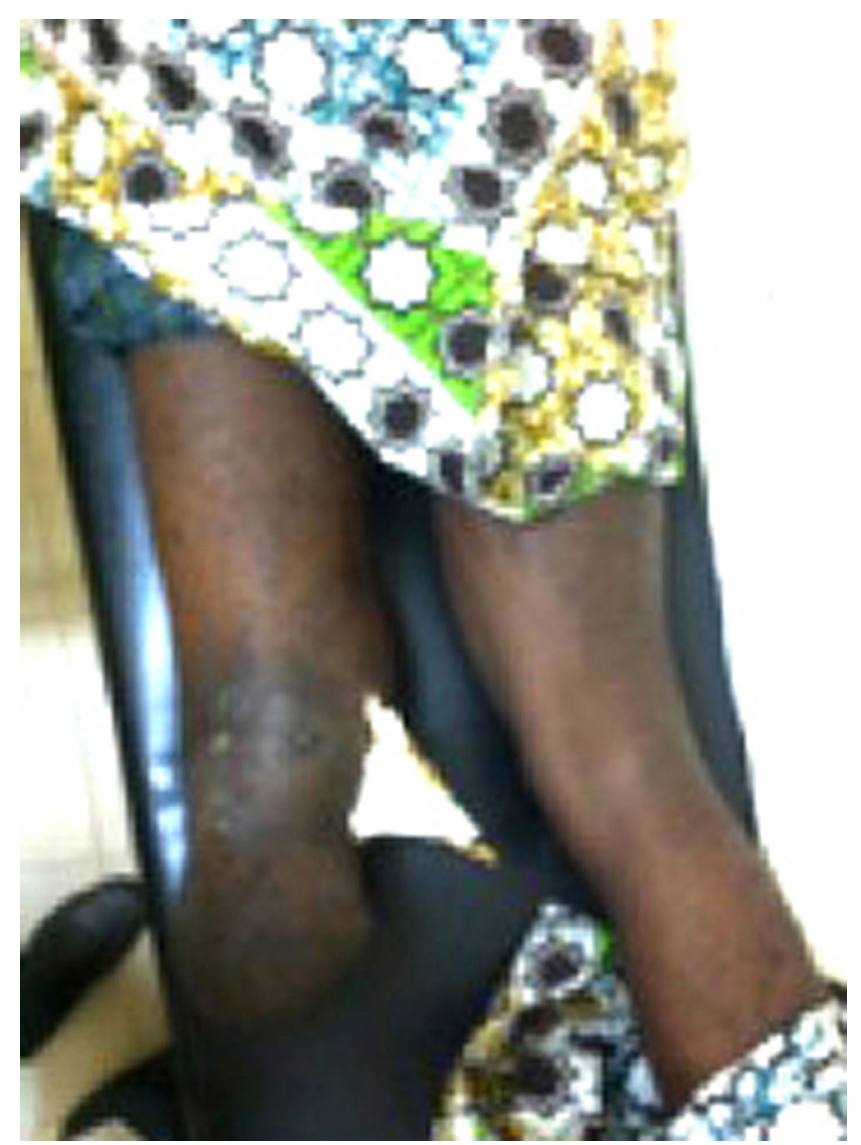

Fig 4 Récidive au niveau du siège du mycétome poplité après amputation avec exérèse / Recurrence at the seat of popliteal mycetoma after amputation with excision

\section{Lavoisier}




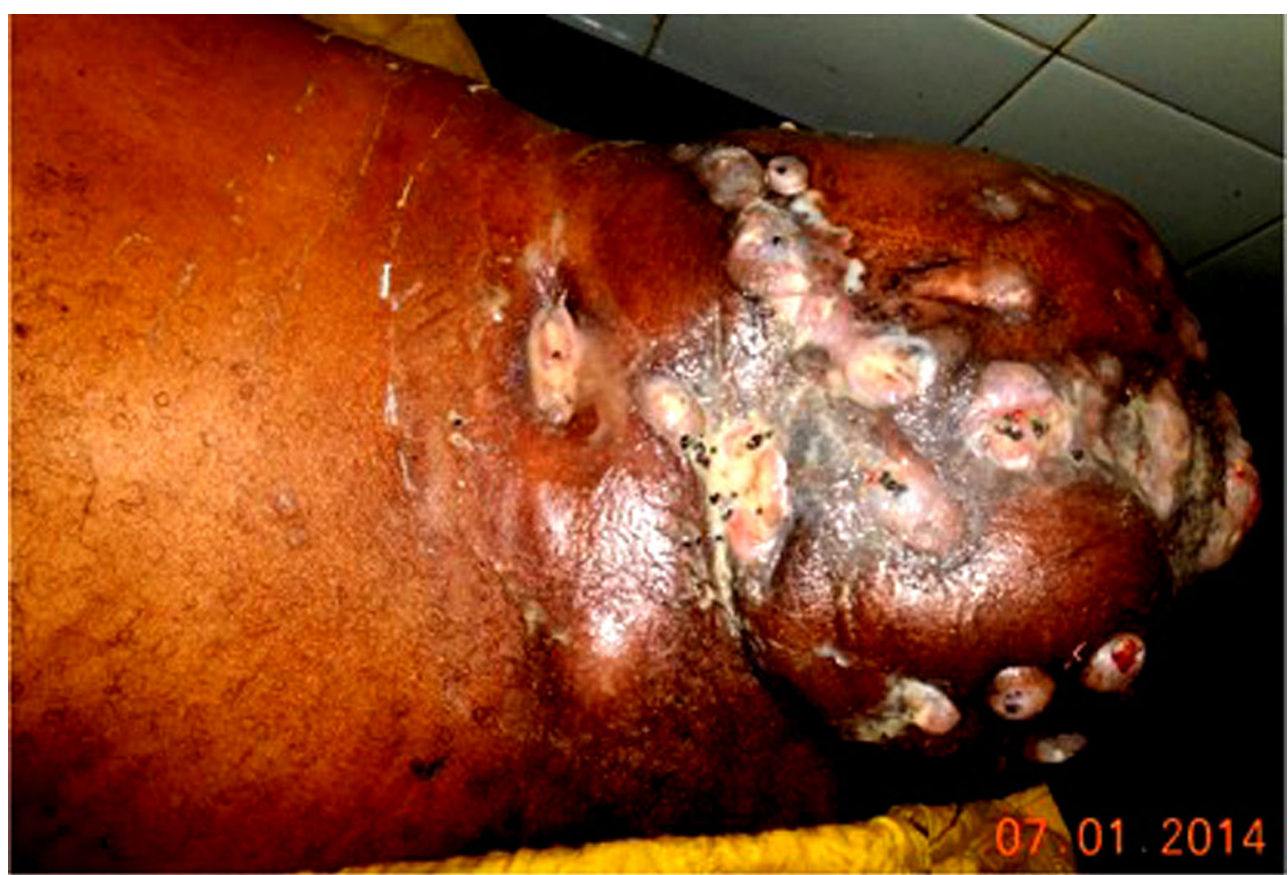

Fig. 5 Récidive de mycétome sur moignon d'amputation de la cuisse / Recurrence of mycetoma on amputation stump of the thigh

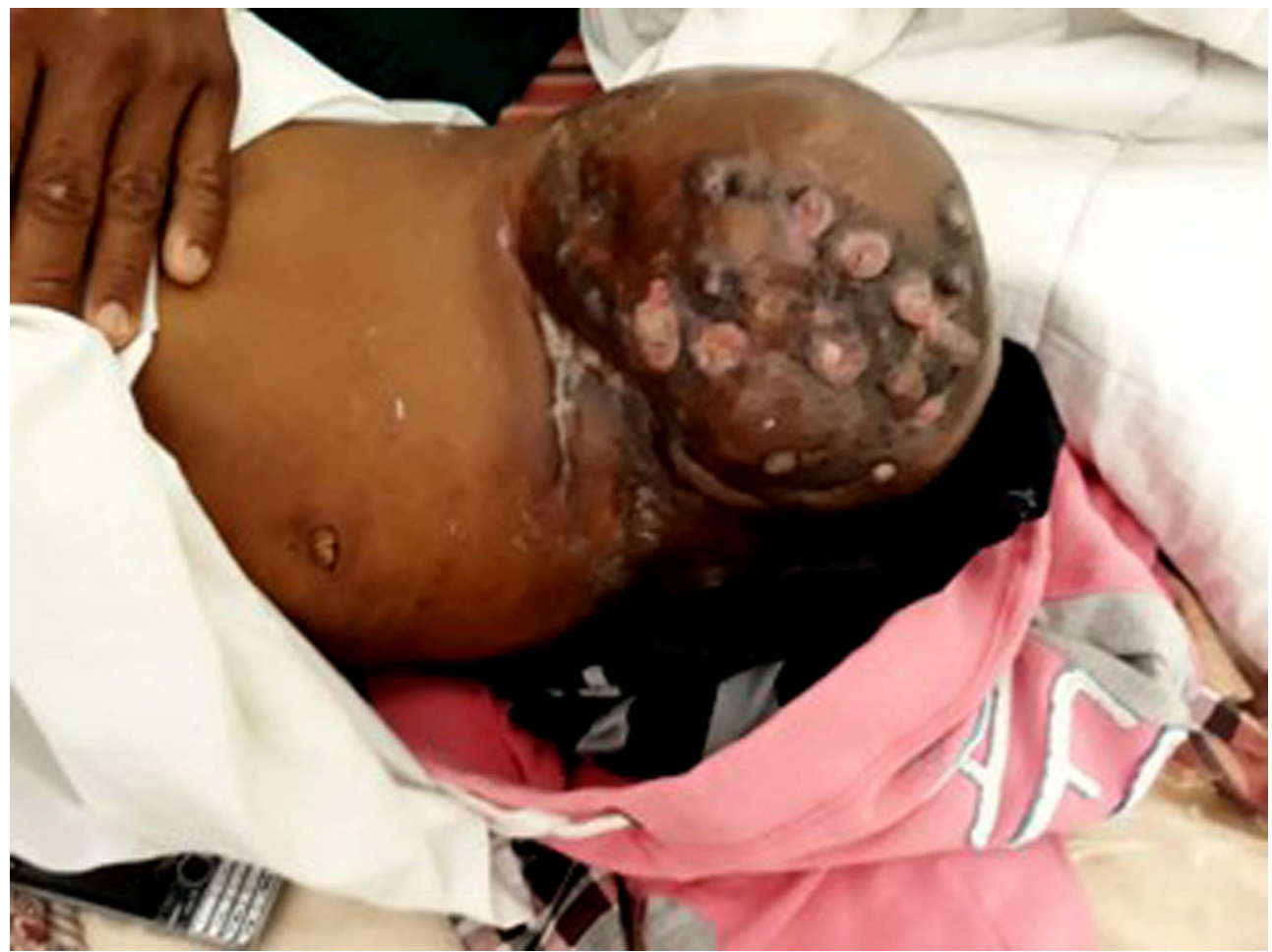

Fig. 6 Multiples fistules au niveau du moignon d'amputation et inguinale / Multiple fistulas at the level of the amputation and inguinal stump. 


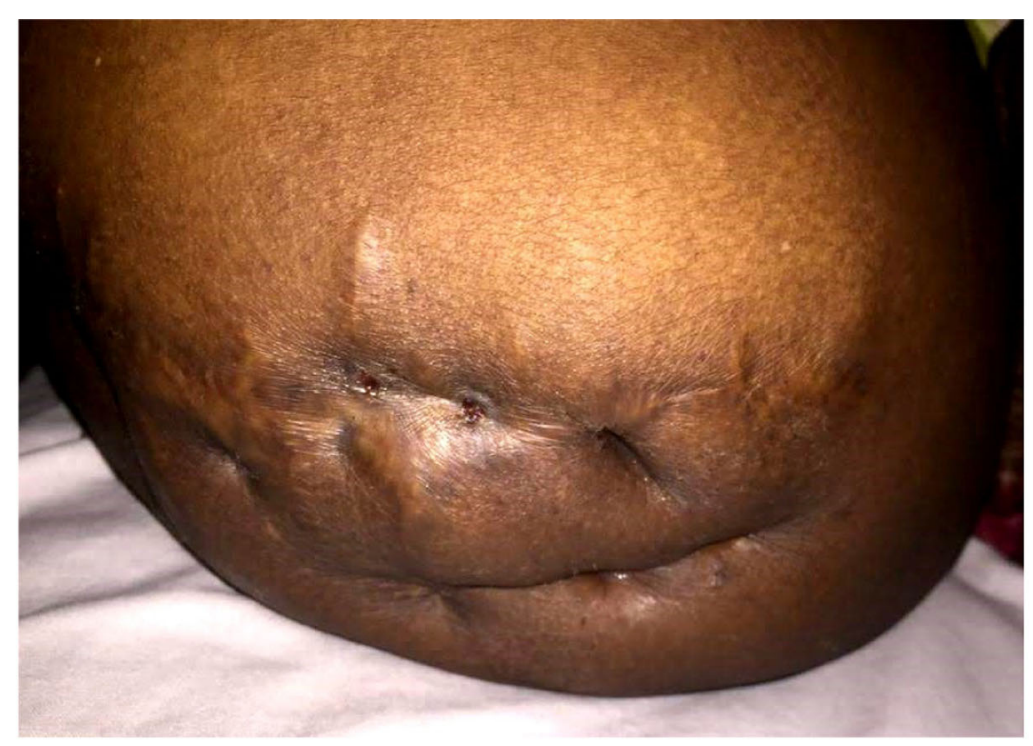

a)

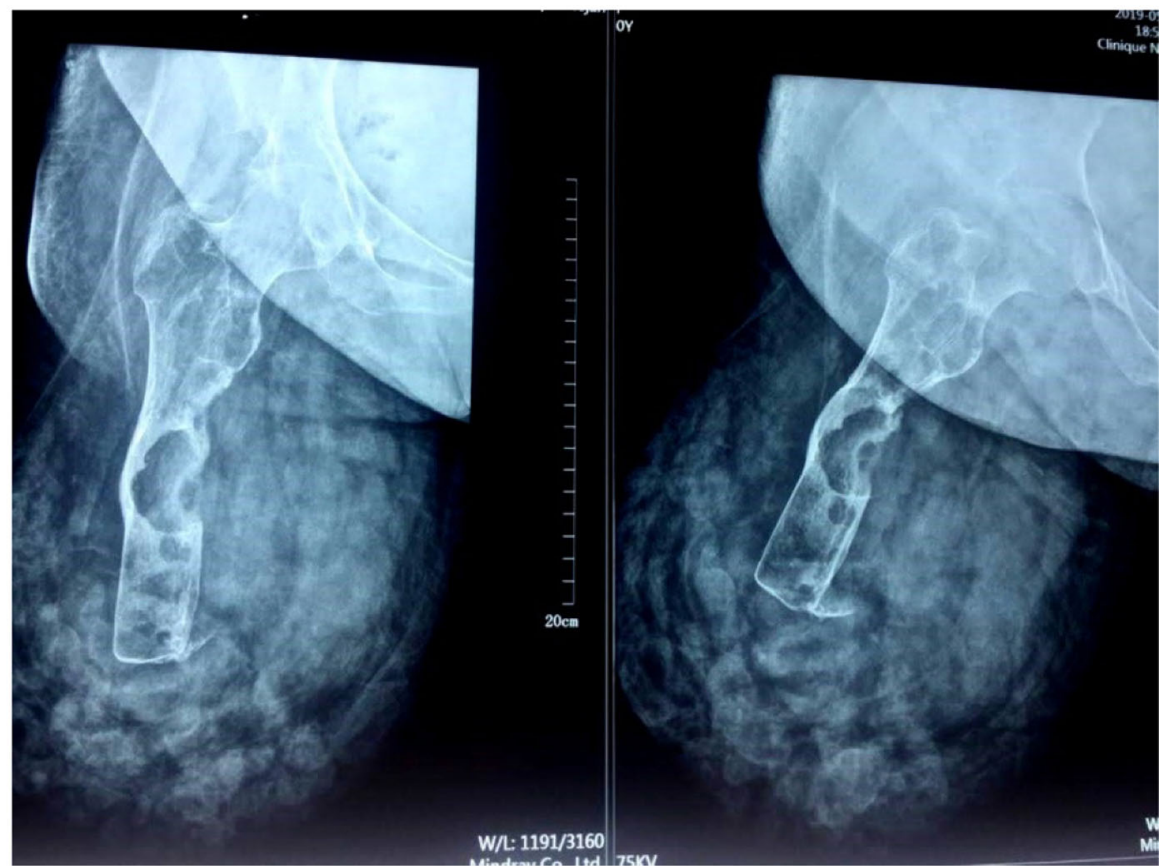

b)

Fig. 7 Fistules productives avec issue de grains noirs sur la cicatrice opératoire avec importante ostéolyse sur le moignon restant du fémur / Productive fistulas with black grains production on chirurgical scar with major osteolysis on the remaining femur stump

présentait de multiples fistules sur la cicatrice du moignon avec issue de grains noirs. L'imagerie (radiographie, scanner) a permis de mettre en évidence l'importance de l'atteinte osseuse avec présence de lacunes à l'emporte-pi èce. Une indication de désarticulation de hanche avec curage ganglionnaire inguinal a été retenue mais refusée par la patiente.

\section{Commentaires}

Le taux de récidive de mycétome après traitement est élevé. Il varie de $18 \%$ à $50 \%$ [2,5-7]. Les récidives après amputation ne sont pas rares. Leur prévalence n'est pas connue, puisque nous n'avons pas retrouvé d'articles qui s'intéressent exclusivement à ce sujet. Nous avions noté 2 cas sur

\section{Lavoisier}


21 amputations [5] dans notre étude, pour un recul moyen de 25,5 mois et 18 cas sur 51 amputations soit 35,3\% pour Wadal [7].

Les causes de ces récidives sont multifactorielles. Le niveau de l'amputation est un facteur essentiel dans la survenue de ces récidives. Pour ces mycétomes fongiques de traitement chirurgical carcinologique, le site d'amputation doit se faire en zone saine. L'analyse du scanner d'un de nos patients montre qu'il existait des skip métastases et qu'une désarticulation de hanche aurait été meilleure. Il en est de même du patient où la récidive a été notée au niveau du site de l'exérèse. Une amputation de cuisse aurait été préférable pour ce patient. Les patients demandent souvent à avoir le moignon le plus long possible, ce qui parfois influence le site d'amputation et peut être à l'origine de récidive. Ce d'autant plus qu'en cas de récidive, ils refusent un nouveau geste chirurgical et ne reviennent à l'hôpital qu'à une période où tout geste avec marge saine devient impossible.

La technique chirurgicale est aussi importante lors de ces amputations. Certaines adénopathies correspondent à des localisations secondaires. Le curage ganglionnaire premier peut ensemencer le site d'amputation ou être le point de départ d'une extension de la maladie.

Nous n'avons pas utilisé d'antifongique en préopératoire du fait du prix de ce traitement et de l'absence de système de sécurité sociale ou de programme national pour encadrer le traitement de cette pathologie. Le protocole rapporté par le Gezira Mycetoma Center [4] du Nord Soudan consiste en l'utilisation de comprimés d'itraconazole en postopératoire pendant 3 mois pour les mycétomes de petite taille $\leq 5 \mathrm{~cm}$ et 6 mois avant et après l'opération pour les lésions de taille moyenne et grande $\geq 5 \mathrm{~cm}$. D'autres protocoles sont rapportés, notamment par le Mycetoma Medical Center de Khartoum [6]. Le traitement antifongique permettrait de mieux circonscrire les lésions afin de faciliter la chirurgie. On ignore par contre sa capacité à diminuer le risque de récidive après chirurgie. Fahal [3] retrouve un taux de récidive de $30 \%$ après chirurgie associée à un traitement médical antifongique.

Selon Develoux [1], il n'existe pas de critère de guérison. Il est donc important de pouvoir suivre les patients amputés sur plusieurs années pour pouvoir mieux apprécier l'apport de l'amputation dans le traitement des mycétomes fongiques avec atteinte osseuse.

\section{Conclusion}

Une bonne planification préopératoire permet une optimisation du geste opératoire et d'éviter certaines récidives.

Liens d'intérêts Les auteurs déclarent ne pas avoir de liens d'intérêts.

\section{Références}

1. Develoux M (2016) Les mycétomes et leur traitement. J Mycol Med 26(2):77-85. doi: 10.1016/j.mycmed.2016.03.005. Epub 2016 May 31.

2. Develoux M, Dieng MT, Kane A, Ndiaye B (2003) Prise en charge des mycétomes en Afrique de 1'Ouest. Bull Soc Pathol Exot 96(5):376-82 [http://www.pathexo.fr/documents/articlesbull/T96-5-2472-DT8.pdf]

3. Fahal A, Mahgoub el S, El Hassan AM, Abdel-Rahman ME (2015) Mycetoma in the Sudan: an update from the Mycetoma Research Centre, University of Khartoum, Sudan. PLoS Negl Trop Dis 27;9(3):e0003679. doi: 10.1371/journal.pntd.0003679. eCollection 2015 Mar

4. Gismalla MDA, Ahmad GMA, Mohamed Ali MM, et al (2019) Surgical management of eumycetoma: experience from Gezira Mycetoma Center, Sudan. Trop Med Health 47: 6. doi: 10.1186/s41182-018-0129-2

5. Sarr L, Niane MM, Diémé CB et al (2016) Chirurgie des mycétomes fongiques à grain noir. À propos de 44 patients pris en charge à l'Hôpital Aristide le Dantec de Dakar (Sénégal) de décembre 2008 à mars 2013. Bull Soc Pathol Exot 109(1):8-12. doi: 10.1007/s13149-015-0463-4. Epub 2015 Dec 28 [http://www. pathexo.fr/documents/articles-bull/2016_109_08.pdf]

6. Suleiman SH, Wadaella el S, Fahal AH (2016) The Surgical Treatment of Mycetoma. PLoS Negl Trop Dis 10(6):e0004690. doi: 10.1371/journal.pntd.0004690. eCollection 2016 Jun

7. Wadal A, Elhassan TA, Zein HA, et al (2016) Predictors of Postoperative Mycetoma Recurrence Using Machine-Learning Algorithms: The Mycetoma Research Center Experience. PLoS Negl Trop Dis 10(10):e0005007. doi: 10.1371/journal.pntd.0005007. eCollection 2016 Oct 